\title{
Thymic lymphoid hyperplasia with Graves' disease in a 28-year- old female: a case report
}

\author{
Kai Zhang ${ }^{1 \#}$, Weibin $\mathrm{Wu}^{1 "}$, Yonghui $\mathrm{Wu}^{1}$, Mingjun Bai ${ }^{2}$, Xiaojun $\mathrm{Li}^{1}$, Jian Zhang ${ }^{1}$, Lijiai Gu ${ }^{1}$, Huiguo Chen ${ }^{1}$ \\ ${ }^{1}$ Department of Thoracic Surgery, ${ }^{2}$ Department of Vascular Interventional Radiology, the Third Affiliated Hospital of Sun Yat-sen University, \\ Guangzhou 510630, China \\ \#These authors contributed equally to this work. \\ Correspondence to: Huiguo Chen. Department of Thoracic Surgery, the Third Affiliated Hospital of Sun Yat-sen University, No. 600 Tianhe Road, \\ Guangzhou 510630, China. Email: andychen820727@hotmail.com.
}

\begin{abstract}
Thymic lymphoid hyperplasia with Graves' disease (GD) is not uncommon in adults. Generally, cases are newly diagnosed with GD when they refer to the department of endocrinology in hospital, and an anterior mediastinal mass is found on a computed tomography scan by accident. Almost half of them receive thymectomy due to the concern about thymoma or thymic carcinoma. In the past literature, an enlarged thymus can gradually shrink after treatment of antithyroid drugs. In this paper, a 28-year-old woman presented to our hospital with a 11-month history of dizziness, left hand convulsion and paralysis, without chest pain, difficulty swallowing, dyspnea. Chest computed tomography revealed an anterior mediastinal mass without obvious nodules. However, in this case, the mass did not shrink obviously after regularly taking antithyroid drugs. In order to figure out the diagnosis of the mass, we performed a thoracoscopic thymic resection, and the pathologic result was thymic lymphoid hyperplasia. There is no thymus gland tissue left on a repeated CT scan four months later after surgery. In this report, we discuss the optimal therapeutic strategy for this rare case. In conclusion, if an anterior mediastinal mass in GD patients did not shrink obviously upon treatment of antithyroid drugs, minimally invasive surgery should be taken into consideration seriously to exclude the possibility of malignancy.
\end{abstract}

Keywords: Thymus; lymphoid hyperplasia; Graves' disease (GD); thymectomy; case report

Submitted Nov 15, 2019. Accepted for publication Dec 18, 2019.

doi: $10.21037 /$ gs.2019.12.18

View this article at: http://dx.doi.org/10.21037/gs.2019.12.18

\section{Introduction}

Thymic lymphoid hyperplasia is characterized with an increased number of lymphoid follicles and germinal centers in the thymus (1), which is associated with several autoimmune diseases such as myasthenia gravis, systemic lupus erythematosus and thymic hyperplasia. We reviewed the literature about Graves' disease (GD)-related thymus enlargement and found 157 cases published. However, it is rarely reported in GD and a clear preoperative diagnosis is difficult. Mendelson et al. revealed that it was difficult to distinguish lymphoid hyperplasia from true hyperplasia just based on imaging features (2). It has been showed that thymic hyperplasia in GD is associated with thyrotropin receptors (TSHR) in thymus tissue. This article presents a case of a 28-year-old young woman with thymic lymphoid hyperplasia associated with GD. The anterior mediastinal mass did not significantly shrink after 2 weeks of treatment with an antithyroid drug (methimazole), and the patient suffered extreme anxiety, so minimally invasive thoracoscopic thymectomy was put into use to make a definitive diagnosis finally.

\section{Case presentation}

A 28-year-old female presented to a local hospital with thyrotoxic symptoms, including exophthalmos, irritability, palpitation, bulimia and weight loss 11 months ago. 

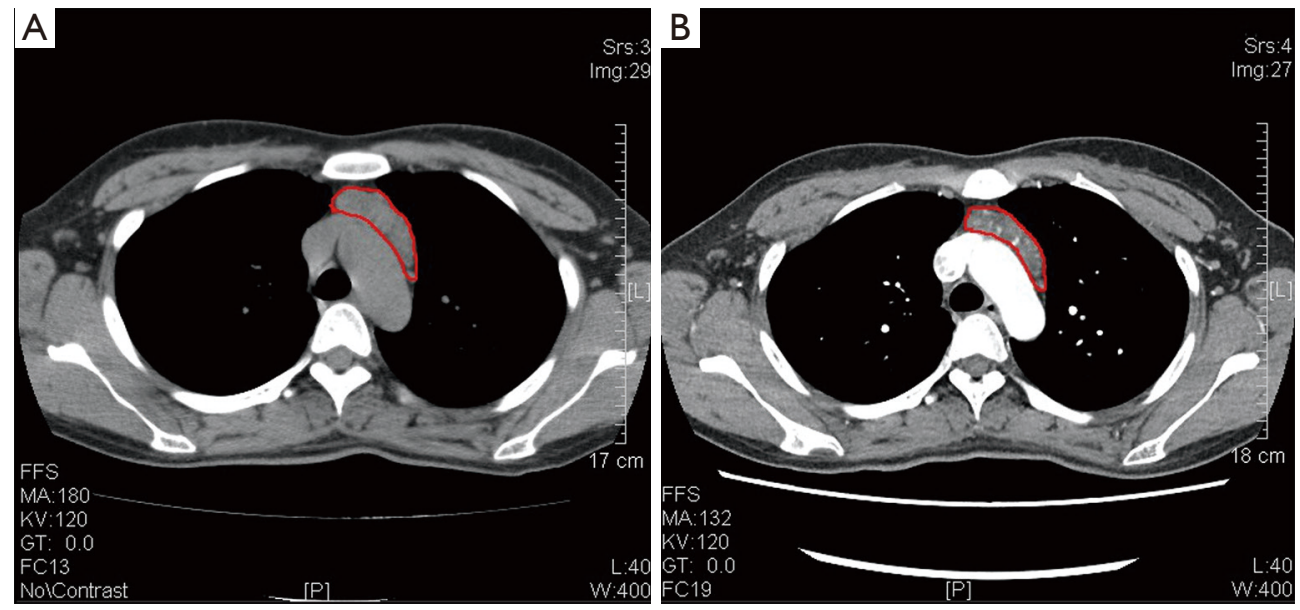

Figure 1 Axial section of chest CT showing an anterior mediastinal mass: pre-treatment $\left(\mathrm{A}, 8.5 \times 6.0 \times 2.1 \mathrm{~cm}^{3}\right)$ versus post-treatment $(\mathrm{B}$, $\left.8.3 \times 5.8 \times 2.0 \mathrm{~cm}^{3}\right)$.

She had no tumor family history, genetic history and psychosocial history. She reported no chest pain, difficulty swallowing, dyspnea and hypokalemic periodic paralysis. Physical examination showed a swelling in the front of neck and exophthalmos. Neck ultrasonography showed an enlarged thyroid gland, which was 2 times as large as the normal without discrete nodules. Her thyroid functions were suggestive of hyperthyroidism at that time: free T4, $105.73 \mathrm{pmol} / \mathrm{L}$ (reference range, $12-22 \mathrm{pmol} / \mathrm{L}$ ); free T3, $54.57 \mathrm{pmol} / \mathrm{L}$ (reference range, 3.1-6.8 pmol/L); thyrotropin (TSH), <0.001 U/L (reference range, $0.27-$ $4.20 \mu \mathrm{IU} / \mathrm{mL}$ ). Based on clinical, biochemical and ultrasound appearance, she was diagnosed with GD. A regimen of methimazole $(20 \mathrm{mg}$ per day for 3 months, and then $10 \mathrm{mg}$ per day for 8 months) was initiated to achieve euthyroidism. After an initial medication for nearly 11 months, above associated symptoms were relieved notably. However, a computed tomography (CT) scan was not performed to evaluate the mediastinum.

On December 19th in 2018, the young woman was further referred to our hospital complaining of severe dizziness, left hand convulsion and paralysis. In order to figure out the cause, chest CT scan was performed immediately. Signs of an unexpected anterior mediastinal mass were found (Figure 1A). Meanwhile, laboratory testing turned out to be euthyroid: free T4, $13.63 \mathrm{pmol} / \mathrm{L}$ (reference range, 9.01-19.05 pmol/L); free T3, $4.92 \mathrm{pmol} / \mathrm{L}$ (reference range, $2.63-5.70 \mathrm{pmol} / \mathrm{L}$ ); TSH, $<0.001 \mu \mathrm{IU} / \mathrm{mL}$ (reference range, $0.35-4.94 \mu \mathrm{IU} / \mathrm{mL}$ ); and TRAb (thyrotropin receptor antibody), $3.2 \mathrm{U} / \mathrm{L}$ (reference range, $0-12 \mathrm{U} / \mathrm{L}$ ).
Her antithyroid medication was at a methimazole dose of $10 \mathrm{mg} /$ day without obvious drug side effects. After 2 weeks, a repeated CT scan was carried out for further evaluation. Disappointingly, the size of the thymus was not obviously diminished (Figure 1B). Because thymoma or thymic cancer was not ruled out, and there was no clear surgical contraindications, the patient underwent complete resection of thymus using a 2-port video-assisted thoracoscopic surgery (VATS). The resected specimen measured approximately $8.4 \times 5.5 \times 2.0 \mathrm{~cm}^{3}$ and weighed about $130 \mathrm{~g}$ (Figure 2). And the anterior mediastinal mass proved to be thymic lymphoid hyperplasia. There was normal cortical and medullary differentiation with plentiful lymphoid follicles. Staining for CD20 and CD3 showed a normal distribution of $\mathrm{B}$ cells and $\mathrm{T}$ cells. Immunohistochemistry for epithelial cell cytokeratin was positive. Low-power (40×) view of the thymus tissue [hematoxylin-eosin (HE) staining] shows diffuse lymphoid follicles with multiple germinal centers and greater-power (200x) view shows germinal center in thymic medulla (Figure 3). No adverse effects occurred during the perioperative period. There is no thymus gland tissue left on a repeated CT scan during the postoperative follow-up (nearly 4 months). A timeline succinctly showed the whole medical procedure of the special case (Figure 4).

\section{Discussion}

Anterior mediastinal masses are uncommonly encountered in the clinical work. It is full of challenge to get a welldefined differential diagnosis including thymic hyperplasia, 
thymoma, thymic carcinoma, retrosternal thyroid, lymphoma and metastasis. In general, thymic hyperplasia may occurs as a reactive hyperplasia in multiple systemic disease and is expected to reduce with conservative

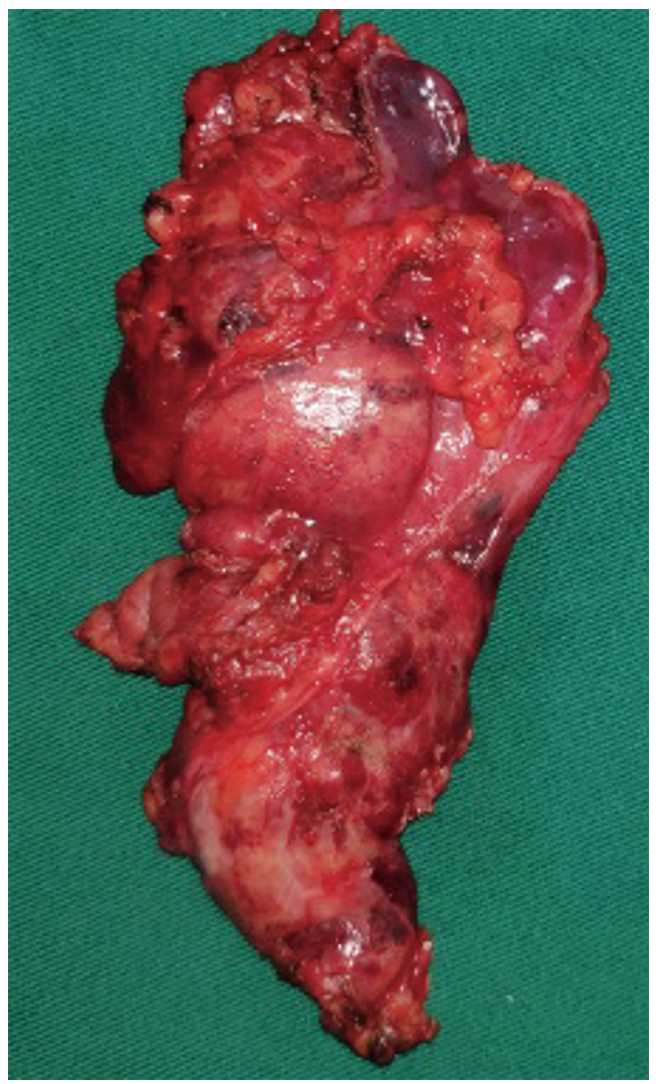

Figure 2 The resected thymic mass enlarges obviously and adheres closely to the surrounding tissue. management.

Thymic hyperplasia is a kind of reversible change associated with GD, which includes two sub-types: true hyperplasia and lymphoid hyperplasia. Thymic lymphoid hyperplasia is regarded as a benign process, and it is consistent with the CT appearances of a homogeneous mass without invasion and associated with a variety of autoimmune diseases. The rate of thymic lymphoid hyperplasia accompanying GD remains unknown, because a CT scan is not routinely performed in GD patients and the diagnosis of thymic lymphoid hyperplasia cannot be easily made just by imaging. This sub-type is mostly related with myasthenia gravis, but recently it has been observed in other autoimmune diseases.

Several studies have reported spontaneous resolution of GD-associated thymic enlargement upon the achievement of a euthyroid state (3). T3 may be associated with thymic hyperplasia (4). It is attributed to that antithyroid drugs might cause a block of thyroid hormone synthesis and induce the involution of thymus by immunosuppressive effects (5), but the precise mechanisms of thymic hyperplasia in GD has not yet been well determined. Most of recent literature supports a conservative approach for thymic hyperplasia associated with GD. However, Levy et al. reported that one patient had type I malignant thymoma associated with GD (6), and we found that the size of anterior mediastinal mass in this case did not significantly decrease in spite of keeping in a state of euthyroid, so the thoracoscopic thymectomy was performed to evaluate the mass. And the patient was satisfied with the operation, because the pathology result ruled out thymic cancer or thymoma and eliminated her fear.

At present, there is no standard processing strategy of
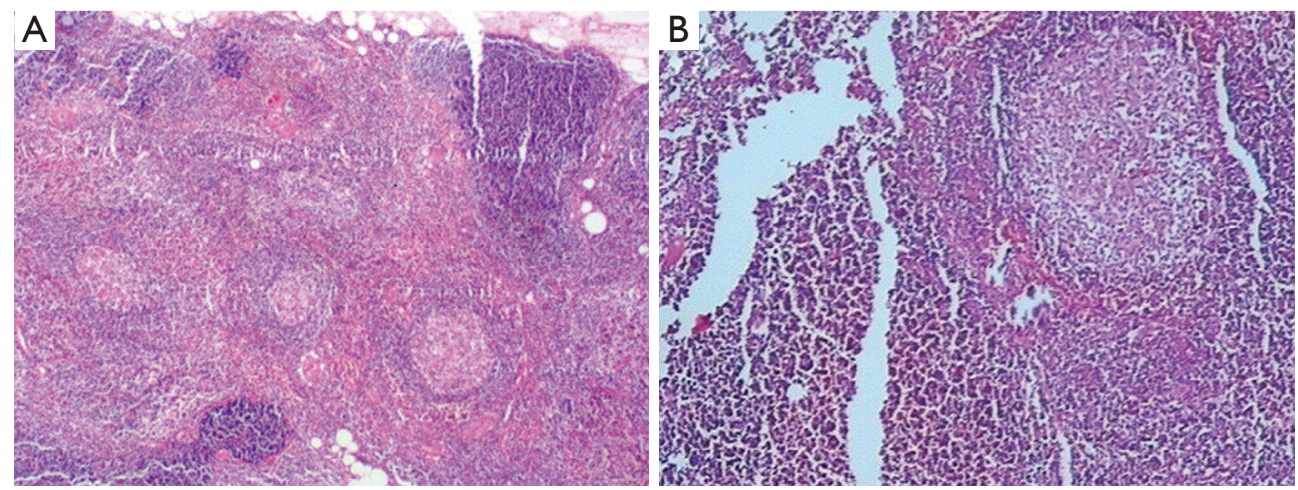

Figure 3 Low-power $(40 \times)$ view of the thymus tissue [hematoxylin-eosin (HE) staining] shows diffuse lymphoid follicles with multiple germinal centers (A). Greater-power (200x) view shows germinal center in thymic medulla (B). 


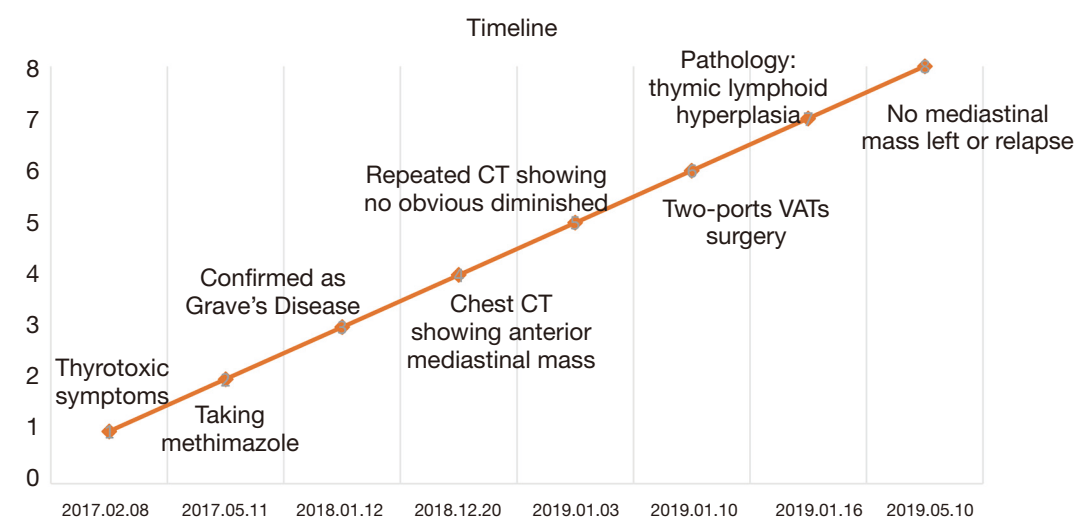

Figure 4 A timeline showing the medical procedure of the case.

thymic lymphoid hyperplasia in GD. Steroid therapy has been proven to be noneffective (7). Generally, surgical interventions are deemed to be unnecessary because the thymic mass can gradually decrease through oral antithyroid drugs. If an anterior mediastinal mass in a GD patient is not significantly reduced after the treatment of antithyroid drugs, what are we supposed to do? In our opinion, minimal invasive procedure, such as thoracoscopic thymectomy, should be taken into consideration under the circumstances. Thoracoscopic thymectomy could differentiating benign and malignant mass without delay. And proved benign hyperplasia could eliminate the patient's anxiety compared with traditional long-term follow-up. However, the management of GD associated with anterior mediastinal mass is full of challenge and requires a multi-discipline team for the final diagnosis and treatment.

We believe that all anterior mediastinal masses need a comprehensive assessment, especially for reversible thymic enlargement, and that surveillance is supposed to be considered in some patients prior to surgical removal. We reported an extremely rare case of thymic lymphoid hyperplasia with GD who underwent thymus resection. In conclusion, thymic hyperplasia was closely related to GD. Thymectomy should be considered to exclude the malignancy if an anterior mediastinal mass in GD patients did not shrink obviously upon treatment of antithyroid drugs. A large-scale, multi-center randomized controlled trial is needed to explore the optimal treatment method.

\section{Acknowledgments}

Funding: None.

\section{Footnote}

Conflicts of Interest: All authors have completed the ICMJE uniform disclosure form (available at http://dx.doi. org/10.21037/gs.2019.12.18). The authors have no conflicts of interest to declare.

Ethical Statement: The authors are accountable for all aspects of the work in ensuring that questions related to the accuracy or integrity of any part of the work are appropriately investigated and resolved. Written informed consent was obtained from the patient for publication of this Case report and any accompanying images.

Open Access Statement: This is an Open Access article distributed in accordance with the Creative Commons Attribution-NonCommercial-NoDerivs 4.0 International License (CC BY-NC-ND 4.0), which permits the noncommercial replication and distribution of the article with the strict proviso that no changes or edits are made and the original work is properly cited (including links to both the formal publication through the relevant DOI and the license). See: https://creativecommons.org/licenses/by-nc-nd/4.0/.

\section{References}

1. Shimosato Y, Mukai K, Matsuno Y. Tumors of the mediastinum: atlas of tumor pathology. Armed Forces Institute of Pathology 1997;21:158-68.

2. Mendelson DS. Imaging of the thymus. Chest Surg Clin N Am 2001;11:269-93, x.

3. Haider U, Richards P, Gianoukakis AG. Thymic Hyperplasia Associated with Graves' Disease: 
Pathophysiology and Proposed Management Algorithm. Thyroid 2017;27:994-1000.

4. Jinguji M, Nakajo M, Nakajo M, et al. Thymic Involution After Radioiodine Therapy for Graves Disease:

Relationships With Serum Thyroid Hormones and TRAb. J Endocr Soc 2017;1:852-60.

5. Weetman AP, McGregor AM, Hall R. Evidence for an effect of antithyroid drugs on the natural history of Graves' disease. Clin Endocrinol (Oxf) 1984;21:163-72.

6. Levy Y, Afek A, Sherer Y, et al. Malignant thymoma associated with autoimmune diseases: a retrospective study and review of the literature. Semin Arthritis Rheum 1998;28:73-9.

7. Nasseri F, Eftekhari F. Clinical and radiologic review of the normal and abnormal thymus: pearls and pitfalls. Radiographics 2010;30:413-28.
Cite this article as: Zhang $\mathrm{K}, \mathrm{Wu} \mathrm{W}, \mathrm{Wu} \mathrm{Y}$, Bai $\mathrm{M}, \mathrm{Li} \mathrm{X}$, Zhang J, Gu L, Chen H. Thymic lymphoid hyperplasia with Graves' disease in a 28-year-old female: a case report. Gland Surg 2020;9(2):437-441. doi: 10.21037/gs.2019.12.18 